\title{
Craniopharyngioma arising in a Rathke's cleft cyst: case report
}

\author{
Ahmed K. Alomari, MD, ${ }^{1}$ Brian J. Kelley, MD, PhD, ${ }^{2}$ Eyiyemisi Damisah, MD, ${ }^{2}$ Asher Marks, MD, ${ }^{3}$ \\ Pei Hui, MD, PhD, ${ }^{1}$ Michael DiLuna, MD, ${ }^{2}$ and Alexander Vortmeyer, MD, PhD ${ }^{1}$
}

Departments of ${ }^{1}$ Pathology, ${ }^{2}$ Neurosurgery, and ${ }^{3}$ Pediatrics, Yale University, School of Medicine, New Haven, Connecticut

\begin{abstract}
Craniopharyngioma is one of the most common non-glial intracranial tumors of childhood. Its relation to Rathke's cleft cyst (RCC) is controversial, and both lesions have been hypothesized to lie on a continuum of cystic ectodermal lesions of the sellar region. The authors report on a 7-year-old boy who presented with decreased visual acuity, presumably of at least 2 years' duration, and was found to have a 5.2-cm sellar lesion with rim enhancement. Histological examination of the resected lesion showed a mixture of areas with simple RCC morphology with focal squamous metaplasia and areas with typical craniopharyngioma morphology. Immunohistochemical staining with CK20 and Ki 67 differentially highlighted the 2 morphological components. Testing for beta-catenin and BRAF mutations was negative in the craniopharyngioma component, precluding definitive molecular classification. Follow-up imaging showed minimal residual enhancement and the patient will be closely followed up with serial MRI. Given the clinical and histological findings in the case, a progressive transformation of the RCC to craniopharyngioma seems to be the most plausible explanation for the co-occurrence of the 2 lesion types in this patient. An extensive review of previously proposed theories of the relationship between craniopharyngioma and RCC is also presented.
\end{abstract}

http://thejns.org/doi/abs/10.3171/2014.11.PEDS14370

KEY WORDS craniopharyngioma; Rathke's cleft cyst; BRAF; beta-catenin; oncology

$\mathrm{C}$ YSTIC lesions of the sellar and parasellar regions represent a nontrivial proportion of intracranial space-occupying lesions. Within this group, craniopharyngiomas are the most common non-glial tumors of childhood but remain relatively rare when examined on a population basis. ${ }^{16}$ These locally aggressive neoplasms have cellular pathology that arises from squamous epithelial cell rests occurring anywhere along the craniopharyngeal duct, the canal that connects the stomodeal ectoderm with the invaginating Rathke's pouch..$^{12,19}$ Epidemiological studies indicate a bimodal distribution (preteens and older adults), with the adamantinomatous cell-type variant being found in all ages while a papillary subtype is predominantly found in adults. ${ }^{13}$ In contrast, symptomatic Rathke's cleft cyst (RCC) is a less common lesion and represents a separate entity composed of benign enlarging remnants of the craniopharyngeal duct or Rathke's pouch. ${ }^{19}$

The suggestion that these ectodermal lesions potentially represent a continuum has gained more popularity over the last 2 decades in an attempt to explain the occurrence of rare lesions with mixed or ambiguous features, with most information coming in the form of case reports or small case series. ${ }^{7,9,10,12}$ Herein, we present a unique case of craniopharyngioma admixed with histologically unequivocal RCC in a 7-year-old boy along with a literature review of the theories that have been proposed with respect to the relationship between craniopharyngioma and RCC.

\section{Case Report}

History and Examination

This 7-year-old right-handed boy presented to neurosurgical attention following approximately 2 years of progressive decrease in visual acuity. His birth history was notable for left gaze nystagmus, to which his poor left eye vision was attributed. His review of systems was notable for polydipsia with no associated polyuria. Review of the patient's pediatric records indicated he was in the 50th percentile for height and weight with a normal serum glucose level. His development was Tanner Stage 1, with nor-

ABBREVIATIONS CK20 = cytokeratin 20; IHC = immunohistochemistry; PCR = polymerase chain reaction; RCC = Rathke's cleft cyst; SSCP = single-strand conformation polymorphism.

SUBMITTED July 22, 2014. ACCEPTED November 4, 2014.

INCLUDE WHEN CITING Published online January 2, 2015; DOI: 10.3171/2014.11.PEDS14370.

DISCLOSURE The authors report no conflict of interest concerning the materials or methods used in this study or the findings specified in this paper. 
mal energy levels and hair pattern. His history revealed no noted personality changes or difficulty with school performance. On physical examination, he was alert and oriented with bilaterally symmetrical pupil reactivity, normal extraocular muscle function, symmetrical smile and palate elevation without uvula deviation, and midline tongue protrusion. The results of motor, sensory, and cerebellar function evaluations were unremarkable. Ophthalmological testing demonstrated decreased visual acuities of 20/60 and 20/400 in the right and left eyes, respectively, along with temporal field deficits that were greater in the right eye than in the left. Additionally, he was unable to fixate with his left eye. Funduscopic examination revealed optic disc pallor, more pronounced on the left side. His visual deficits had been managed using prescription lenses with minimal improvement. A comprehensive endocrinological workup revealed low levels of serum cortisol, FSH/ LH, IGF-1, and free T4 with low-normal ACTH levels, consistent with panhypopituitarism. Serum sodium levels were within normal limits, although urinalysis revealed low osmolality, sodium, and specific gravity, consistent with compensated diabetes insipidus.

\section{Imaging}

Noncontrast head CT revealed a hypodense lesion of approximately $4.5 \times 4.5 \times 5.0 \mathrm{~cm}^{3}$ occupying the sellar and suprasellar regions with associated hyperdensities consistent with calcifications within a cystic lesion (Fig. 1A). Sagittal projections revealed sellar expansion from mass effect with a classic J-shaped appearance. MRI with and without contrast revealed a $4.3 \times 4.4 \times 5.2-\mathrm{cm}^{3} \mathrm{~T} 1$ hypointense and $\mathrm{T} 2$ hyperintense cystic sellar lesion with suprasellar extension and rim enhancement (Fig. 1B). The optic chiasm was displaced superiorly and posteriorly. MR angiography indicated circumferential displacement of the supraclinoid internal cerebral arteries and A1 segments bilaterally (Fig. 1C). There was no restricted diffusion or abnormal vasculature to suggest infection or aneurysm formation.

\section{Operation and Immediate Postoperative Course}

The goals of surgery were to obtain tissue for diagno- sis and to achieve decompression of the optic nerves and optic chiasm via either gross-total resection or subtotal resection with adjuvant radiotherapy. Given the location of the displaced optic chiasm, an open cranial approach was undertaken. In the operating room, the patient was placed supine with Mayfield fixation. Dexamethasone and mannitol were administered perioperatively. A modified right-sided pterional craniotomy was performed, consistent with previous descriptions by Wisoff and Donahue. ${ }^{16}$ Additional brain relaxation was obtained via drainage of cerebrospinal fluid from the basal cisterns. Upon entering the cyst, an oily liquid was encountered. The tumor was removed in a piecemeal fashion because of its size. Residual tumor near the infundibulum could not be visualized and removed safely. Postoperatively, the patient's visual acuity and fields were found to be stable on clinical examination, albeit with some subjective improvement noted by the patient within a few days. His diabetes insipidus and adrenal insufficiency were initially managed with desmopressin and hydrocortisone, respectively, with an eventual transition to intranasal desmopressin and prednisone.

\section{Pathology}

Representative sections were submitted for frozen section examination. The remainder of the specimen was fixed in $10 \%$ buffered formalin and then embedded in paraffin. Four-micrometer sections were cut onto glass slides and placed in a $60^{\circ} \mathrm{C}$ oven for 1 hour. Routine histological examination was performed with $\mathrm{H} \& \mathrm{E}$ staining. Immunohistochemical analysis included antibodies against beta-catenin, cytokeratin 20 (CK20), Ki 67 (Dako Corporation), and BRAF (Spring Bioscience) used at dilutions suggested by the manufacturer. Evaluation for the presence of the BRAF V600E mutation was also attempted following manual microdissection. Polymerase chain reaction (PCR) amplification of the target gene was performed, followed by assessment for single-strand conformation polymorphism (SSCP).

Representative H \& E-stained sections showed small complex cystic spaces and an infiltrative stratified squamous epithelium without a palisading basal layer. Abundant "wet" keratin, calcifications, and a marked inflam-
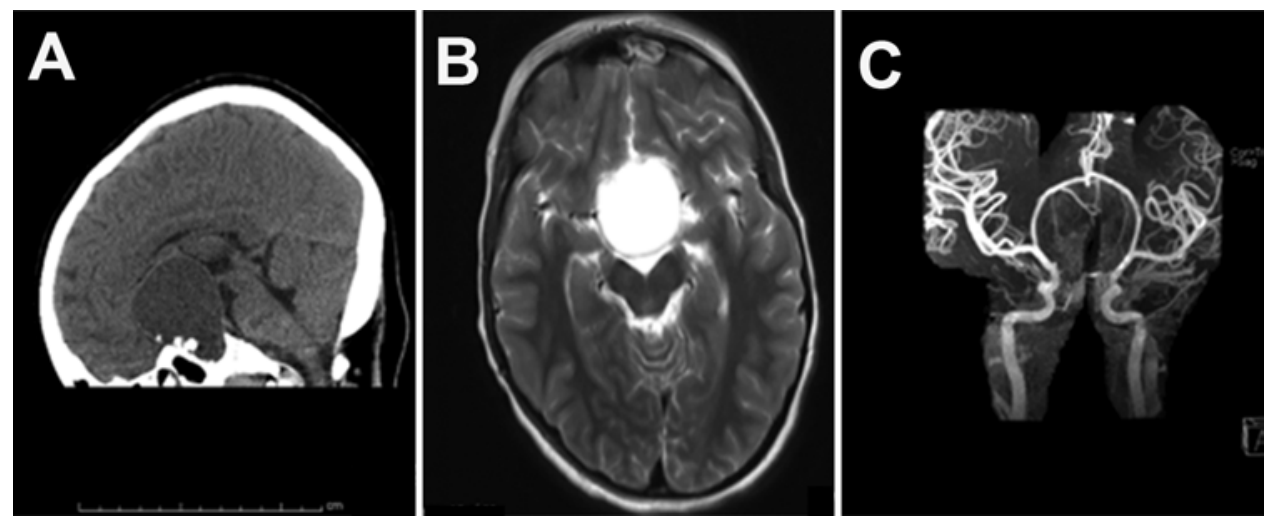

FIG. 1. A: Sagittal noncontrast CT scan demonstrating a $4.5 \times 4.5 \times 5.0-\mathrm{cm}^{3}$ sellar and suprasellar mass with small hyperdensities, consistent with calcification, and a largely hypodense cystic core. B: Axial T2-weighted MR image demonstrating a T2 hyperintense cystic sellar lesion. C: MR angiogram demonstrating circumferential bilateral displacement of the supraclinoid internal cerebral arteries and first segment of the anterior cerebral arteries. 
matory infiltrate were also identified. These findings were consistent with craniopharyngioma pathology (Fig. 2). Most of the specimen, however, was composed of numerous cystic spaces lined by simple, partially ciliated columnar epithelium surrounded by mild fibrosis without evidence of calcification, keratin formation, or a significant inflammatory infiltrate. These findings were consistent with RCC (Fig. 3A). Additionally, areas with intermediate histological features of cystic spaces lined by metaplastic squamous-like epithelium without calcification along with minimal keratin formation were recognized (Fig. 3B). Definitive transition between the RCC and the craniopharyngioma components was not identified, likely due to the highly fragmented nature of the specimen.

Representative sections stained with beta-catenin antibody showed membranous and cytoplasmic immunoreactivity throughout the craniopharyngioma epithelium with no nuclear staining (Fig. 2D), while BRAF immunohistochemistry (IHC) showed only focal faint positivity in the epithelial cells. Additional IHC included staining for CK20, which highlighted both the simple epithelium of the Rathke's cyst and the metaplastic squamous epithelium but did not stain the invasive craniopharyngioma epithelium (Fig. 3C). Ki 67 reactivity demonstrated a higher labeling index in the metaplastic squamous epithelium and invasive epithelium of the craniopharyngioma when compared with simple Rathke's cyst epithelium (Fig. 3D). The BRAF V600E mutation was not detected using PCRSSCP in selectively dissected sections of craniopharyngioma on repeated testing.

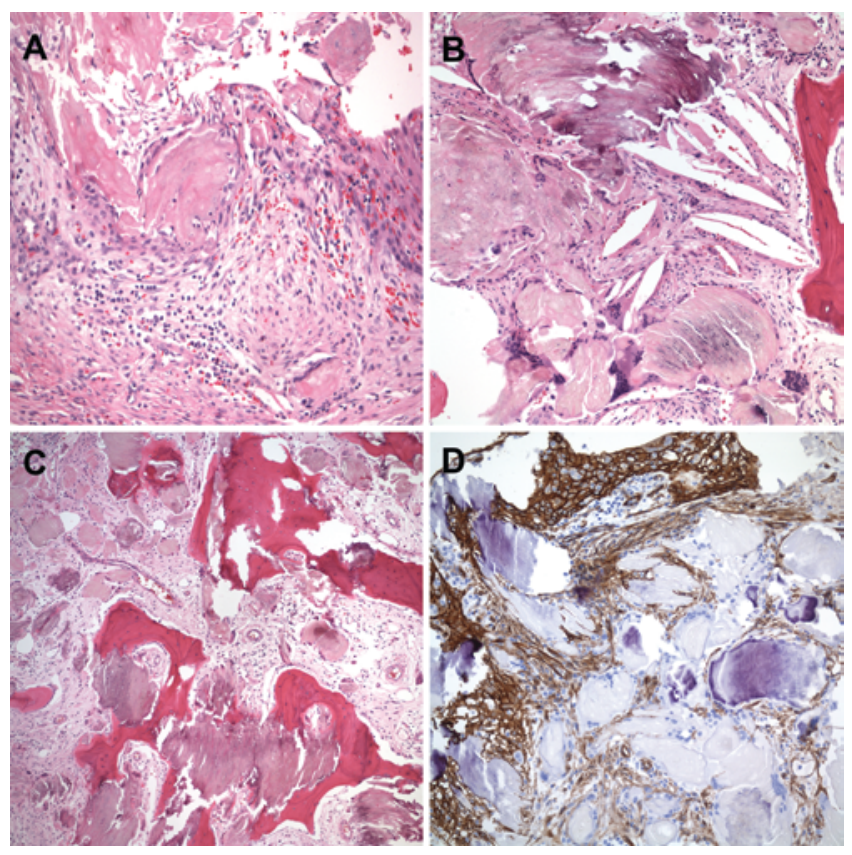

FIG. 2. Representative photomicrographs depicting the different morphological features of craniopharyngioma. A: $\mathrm{H}$ \& E-stained section showing squamous epithelium admixed with "wet" keratin and surrounded by chronic inflammatory cells. B and C: $\mathrm{H} \&$ E-stained sections demonstrating cholesterol clefts, "wet" keratin, foreign body giant cell reaction, and classification. D: Section immunostained for beta-catenin showing membranous and cytoplasmic, but not nuclear, positivity in the epithelial cells.
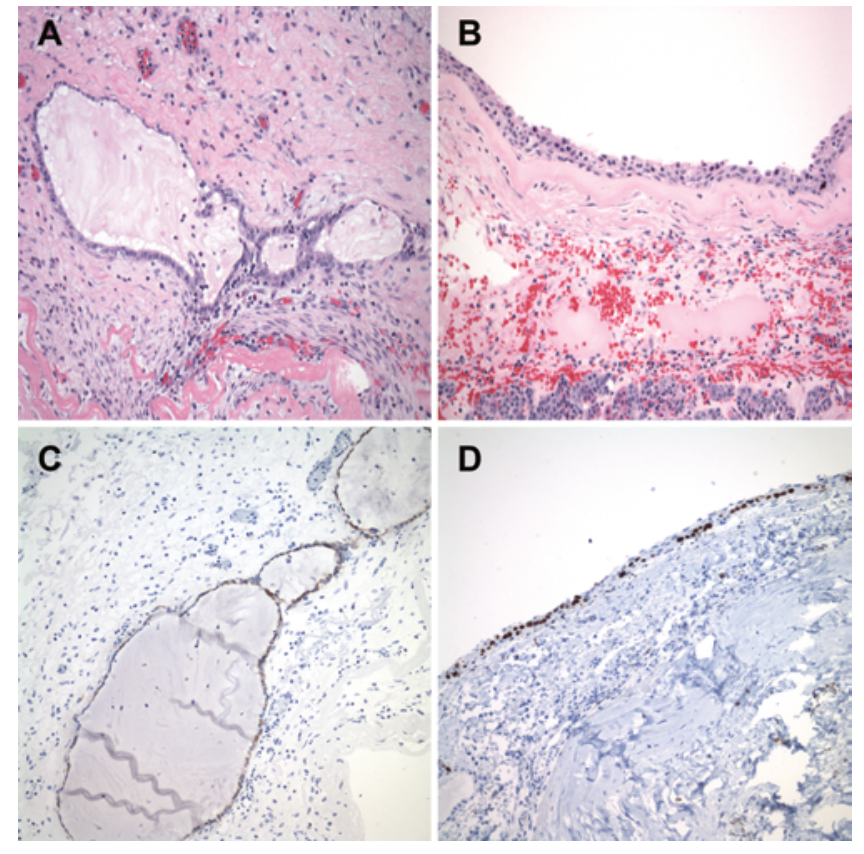

FIG. 3. Representative photomicrographs depicting the different morphological features of RCC. A: H \& E-stained section showing multiple small cysts with simple columnar epithelial lining (cilia were focally present), mild chronic inflammatory infiltrate, and fibrosis. B: H \& E-stained section demonstrating larger cysts with noninvasive metaplastic stratified squamous epithelium without keratin formation or calcification. At the bottom of the image, a thin strand of normal pituitary tissue may be seen. C: Section immunostained for CK20 highlighting the simple epithelial lining of the RCC in addition to the metaplastic stratified epithelium (not shown). D: Section immunostained for Ki 67 showing a relatively high labeling index in areas of metaplastic squamous epithelium in comparison with areas of simple columnar epithelium (not shown).

\section{Follow-Up}

Postoperative MRI revealed minimal enhancement within the sella, which could have represented residual tumor/cyst or postoperative change. Given the noted predominance of RCC histology as well as the minimal enhancement on follow-up scan, the decision was made to follow the patient closely with MRI every 3 months. Focal radiation therapy will be initiated if there is any evidence of recurrent tumor growth on surveillance imaging.

\section{Discussion}

Craniopharyngiomas represent a relatively small but clinically significant number of pediatric brain tumor diagnoses each year. These neoplasms are traditionally localized within the sellar and parasellar region and have been linked to progression of benign RCC by reports of tumor development after RCC resection. ${ }^{12,14}$ These reports stress the time lapse between initial diagnosis/resection and recurrence in adult patients. Our report is unique in that we discuss comparable findings from a 7-year-old patient who had not undergone any previous surgery or adjuvant therapy. Our histopathological findings are also unique in that the craniopharyngioma component of this mixed tumor showed ambiguous features that precluded definitive classification into adamantinomatous or papil- 
lary subtypes. Taken together, these findings may suggest either a transformative lesion with progression from RCC to craniopharyngioma or development of each lesion independently, given the lack of clear histological transition. In light of our review of the current literature, a progressive transformation seems most plausible.

The current WHO classification identifies 2 variants of craniopharyngioma; the adamantinomatous variant is characterized by invasive stratified epithelium with palisading basal cells admixed with "wet" keratin, calcifications, chronic inflammation, and cholesterol clefts, whereas the papillary variant is characterized by invasive stratified squamous epithelium-lined papillae with prominent fibrovascular cores, usually lacking significant calcification and keratin formation. ${ }^{13}$ By comparison, histological examination of RCCs usually shows cysts lined by simple ciliated cuboidal to columnar cells with occasional goblet cells. The occasional presence of squamous metaplasia is well recognized.15 The differentiation between these RCCs and craniopharyngiomas is of great clinical significance for optimum preoperative planning to include the extent of resection in addition to the distinct outcome results between the 2 lesions. ${ }^{12}$ Craniopharyngiomas tend to infiltrate adjacent structures and have high reported recurrence rates of more than 25\%. ${ }^{5}$ However, RCC has a much lower tendency to infiltrate adjacent structures and a variable recurrence rate between $11 \%$ and $18 \%$ according to different reports. ${ }^{1}$ Discriminatory radiological criteria have been proposed but showed less than optimum results, with parameters such as the size of the lesion $(>$ $2 \mathrm{~cm}$ ), predominant suprasellar location, and calcification all favoring craniopharyngioma. ${ }^{8}$ Histological examination remains the most reliable and definitive method to differentiate between these 2 lesions, and intraoperative pathology evaluation is crucial to guide management in problematic cases.

Despite these histological classifications, cystic lesions of the parasellar region with ambiguous and overlapping histology have been reported, probably first by Fager and Carter in $1966 .^{6}$ The idea of a spectrum of ectodermally derived cystic lesions in the parasellar region was first proposed by Harrison et al. Interestingly, of the 19 cases of sellar and parasellar epithelial cystic lesions they described, 3 showed atypical histological findings and could not be definitely classified as either RCC or craniopharyngioma.7 Similarly, rare studies have reported cases of RCC with smooth transition from the ciliated columnar epithelium to the stratified squamous epithelium, with calcification more characteristic of craniopharyngioma. ${ }^{10}$ Our case shows similar and more prominent findings, with areas having the classical characteristic of ciliated simple epithelium transitioning to more complex epithelium in addition to areas of invasive well-differentiated stratified squamous epithelium with "wet" keratin and extensive calcifications. The proximity of these different areas to one another, in addition to evidence of transitional histology, argues in favor for the hypothesis that the craniopharyngioma pathology progressed from the benign RCC.

Another lesion with mixed or transitional histology is the so-called "ciliated craniopharyngioma," with less than 20 reported cases in the literature. ${ }^{9,12,14}$ In the majority of these cases, the craniopharyngioma was of the papillary subtype and showed foci of ciliated columnar epithelium, with goblet cells admixed with the tumor. ${ }^{14}$ These lesions were also viewed as evidence of a histogenetic relationship between craniopharyngioma and RCC. ${ }^{11}$

Evidence of such assumption is also supported by cases of subtotal resection of an otherwise classic RCC that recurred after a variable length of time with histological features most consistent with craniopharyngioma. ${ }^{12,17} \mathrm{Ad}-$ ditionally, it has been shown that the presence of squamous metaplasia in cases of RCC influenced their clinical outcome by carrying a higher risk of recurrence similar to that of craniopharyngioma. ${ }^{1}$ Areas of squamous metaplasia showed a higher proliferation index as evidenced by Ki 67 staining when compared with the simple ciliated epithelium. ${ }^{9}$

In a recent report, RCC and papillary craniopharyngioma shared similar staining pattern with pancytokeratin (KL1 clone) and lacked nuclear staining for beta-catenin, while cases of adamantinomatous craniopharyngioma had a significantly different beta-catenin staining profile. ${ }^{8} \mathrm{~A}$ separate study confirmed the exclusive presence of betacatenin mutations in cases of adamantinomatous craniopharyngioma. ${ }^{3}$ However, other studies have shown a distinctive staining profile for cytokeratins 8 and 20 in cases of both craniopharyngioma variants when compared with RCC..$^{18}$ In our case, the distinctive staining pattern for CK20 in portions of the lesion provides further evidence for the coexistence of both entities, a craniopharyngioma and an RCC, in this patient.

In recent years, the results of molecular analysis of craniopharyngioma cases provided additional evidence to support their current clinical and pathological classification. ${ }^{2,3}$ Cases of adamantinomatous craniopharyngiomas harbored mutations in the CTNNBI ( $\beta$-catenin) gene in $60 \%-96 \%$ of patients. ${ }^{2,3}$ These results were confirmed by immunohistochemistry which showed nuclear staining in all cases of adamantinomatous craniopharyngioma while none of the papillary cases showed this finding. ${ }^{2}$ On the other hand, the well-recognized V600E mutation in the BRAF oncogene was identified in $95 \%$ of cases of papillary craniopharyngiomas including rare pediatric cases. ${ }^{2}$ These results were also confirmed by immunohistochemistry. Of note, no other mutation reached statistical significance and the overall somatic mutational burden is small in both subtypes of craniopharyngioma. ${ }^{2}$ In our case, both IHC and molecular testing failed to show convincing evidence of the presence of the $\beta$-catenin or BRAF mutation.

Although a recent meta-analysis of 109 studies and 531 unique cases of craniopharyngioma found that patients who underwent subtotal resection and did not receive radiation therapy had a worse outcome than patients who underwent gross-total resection or those who had subtotal resection and were also treated with radiation therapy, ${ }^{4}$ a decision was made to follow up our patient with serial MRI and to initiate radiation therapy only if there were concerns of recurrence. This was based on the predominance of the RCC histology and the minimal residual enhancement on immediate follow-up imaging.

In summary, we believe that this case represents a rare presentation of craniopharyngioma in a background of 
RCC. Although it is difficult to confirm a temporal relationship between the 2 lesions, the available evidence from our current case and previous similar reports support the hypothesis that the craniopharyngioma progressed from a longstanding RCC via a transitional stage of extensive squamous metaplasia. In light of the recent advancement in our understanding of the molecular pathogenesis of these lesions, it may be possible in the future to better characterize the genetic events that dictate this progression.

\section{References}

1. Aho CJ, Liu C, Zelman V, Couldwell WT, Weiss MH: Surgical outcomes in 118 patients with Rathke cleft cysts. J Neurosurg 102:189-193, 2005

2. Brastianos PK, Taylor-Weiner A, Manley PE, Jones RT, DiasSantagata D, Thorner AR, et al: Exome sequencing identifies BRAF mutations in papillary craniopharyngiomas. Nat Genet 46:161-165, 2014

3. Buslei R, Nolde M, Hofmann B, Meissner S, Eyupoglu IY, Siebzehnrübl F, et al: Common mutations of beta-catenin in adamantinomatous craniopharyngiomas but not in other tumours originating from the sellar region. Acta Neuropathol 109:589-597, 2005

4. Clark AJ, Cage TA, Aranda D, Parsa AT, Sun PP, Auguste KI, et al: A systematic review of the results of surgery and radiotherapy on tumor control for pediatric craniopharyngioma. Childs Nerv Syst 29:231-238, 2013

5. Coffey RJ, Lunsford LD: The role of stereotactic techniques in the management of craniopharyngiomas. Neurosurg Clin N Am 1:161-172, 1990

6. Fager CA, Carter H: Intrasellar epithelial cysts. J Neurosurg 24:77-81, 1966

7. Harrison MJ, Morgello S, Post KD: Epithelial cystic lesions of the sellar and parasellar region: a continuum of ectodermal derivatives? J Neurosurg 80:1018-1025, 1994

8. Hofmann BM, Kreutzer J, Saeger W, Buchfelder M, Blümcke I, Fahlbusch R, et al: Nuclear beta-catenin accumulation as reliable marker for the differentiation between cystic craniopharyngiomas and rathke cleft cysts: a clinico-pathologic approach. Am J Surg Pathol 30:1595-1603, 2006

9. Ikeda H, Yoshimoto T: Clinicopathological study of Rathke's cleft cysts. Clin Neuropathol 21:82-91, 2002

10. Ogawa Y, Tominaga T: A partially ossified solid and cystic Rathke cleft cyst. J Neurosurg 112:1324-1326, 2010

11. Oka H, Kawano N, Yagishita S, Kobayashi I, Saegusa H,
Fujii K: Ciliated craniopharyngioma indicates histogenetic relationship to Rathke cleft epithelium. Clin Neuropathol 16:103-106, 1997

12. Park YS, Ahn JY, Kim DS, Kim TS, Kim SH: Late development of craniopharyngioma following surgery for Rathke's cleft cyst. Clin Neuropathol 28:177-181, 2009

13. Rushing EJ, Giangaspero F, Paulus W, Burger PC: Craniopharyngiomas, in Louis DN Ohgaki $\mathrm{H}$, Wiestler OD, et al (eds): WHO Classification of Tumours of the Central Nervous System, ed 4. Lyon: IARC, 2007, pp 238-240

14. Sato K, Oka H, Utsuki S, Kondo K, Kurata A, Fujii K: Ciliated craniopharyngioma may arise from Rathke cleft cyst. Clin Neuropathol 25:25-28, 2006

15. Voelker JL, Campbell RL, Muller J: Clinical, radiographic, and pathological features of symptomatic Rathke's cleft cysts. J Neurosurg 74:535-544, 1991

16. Wisoff J, Donahue B: Craniopharyngiomas, in Pollack IF, Adelson PD, Albright AL (eds): Principles and Practice of Pediatric Neurosurgery, ed 2. Stuttgart: Thieme, 2008, pp 560-578

17. Wolfe SQ, Heros RC: Editorial. A Rathke cleft cyst to craniopharyngioma: is there a spectrum? J Neurosurg 112:1322-1323, 2010

18. Xin W, Rubin MA, McKeever PE: Differential expression of cytokeratins 8 and 20 distinguishes craniopharyngioma from rathke cleft cyst. Arch Pathol Lab Med 126:1174-1178, 2002

19. Zada G, Lin N, Ojerholm E, Ramkissoon S, Laws ER: Craniopharyngioma and other cystic epithelial lesions of the sellar region: a review of clinical, imaging, and histopathological relationships. Neurosurg Focus 28(4):E4, 2010

\section{Author Contributions}

Conception and design: Vortmeyer, Alomari, Kelley, DiLuna. Acquisition of data: Alomari, Kelley, Damisah, Hui. Analysis and interpretation of data: Alomari. Drafting the article: Alomari, Kelley. Critically revising the article: Vortmeyer, Marks, Hui, DiLuna. Reviewed submitted version of manuscript: all authors. Approved the final version of the manuscript on behalf of all authors: Vortmeyer.

\section{Correspondence}

Alexander Vortmeyer, Neuropathology Program, Department of Pathology, Division of Neuropathology, Yale University School of Medicine, 416A Lauder Hall, 310 Cedar St., New Haven, CT 06520.email: alexander.vortmeyer@yale.edu. 